\title{
Model-based estimation of microscopic anisotropy using diffusion MRI: a simulation study
}

\author{
Andrada Ianuş, ${ }^{\mathrm{a}, *}$, Ivana Drobnjak ${ }^{\mathrm{a}}$, Daniel C. Alexander ${ }^{\mathrm{a}}$ \\ ${ }^{a}$ Center for Medical Image Computing, Department of Computer Science, University College \\ London, Gower Street, London WC1E 6BT, UK
}

\begin{abstract}
Non-invasive estimation of cell size and shape is a key challenge in diffusion MRI. This paper presents a model-based approach that provides independent estimates of pore size and eccentricity from diffusion MRI data. The technique uses a geometric model of finite cylinders with gamma distributed radii to represent pores of various sizes and elongations. We consider both macroscopically isotropic substrates as well as substrates of semi-coherently oriented anisotropic pores and we use Monte Carlo simulations to generate synthetic data. We compare the sensitivity of single and double diffusion encoding (SDE and DDE) sequences to the size distribution and eccentricity and further analyse different protocols of DDE sequences with parallel and/or perpendicular pairs of gradients. We show that explicitly accounting for size distribution is necessary for accurate microstructural parameter estimates, and a model that assumes a single size yields biased eccentricity values. We also find that SDE sequences support estimates, although DDE sequences with mixed parallel and perpendicular gradients enhance accuracy. In the case of macroscopically anisotropic substrates, this model-based approach can be extended to a rotationally invariant framework to provide features of pore shape (specifically eccentricity) in the presence of size distribution and orientation dispersion.
\end{abstract}

Keywords: diffusion MRI, microscopic anisotropy, double pulsed field gradient, pore size distribution, pore eccentricity, compartment models

Word count: 11,037 - including references, title, all numbers and symbols

\footnotetext{
${ }^{*}$ Corresponding author

Email address: a.ianus.11@ucl.ac.uk (Andrada Ianuş)
} 
Abbreviations used: SDE - single diffusion encoding, DDE - double diffusion encoding, FE - fractional eccentricity, MC - Monte Carlo, IFC - isotropic finite cylinders, IGFC - isotropic gamma finite cylinders, WFC - watson finite cylinders, WGFC - watson gamma finite cylinders, CRLB - Cramer-Rao lower bound

\section{Introduction}

Diffusion weighted magnetic resonance imaging (DW-MRI) is a non-invasive probe of molecular displacement, providing structural information at the microscopic level. Cellular membranes in the tissue restrict the diffusion of water molecules so that the measured signal is sensitive to cellular architecture. Accurate estimation of pore size and shape from diffusion data is a key challenge in DW-MRI, with potential applications to cancer imaging to discern differences in tumour microstructure $[1,2]$, white matter imaging to map axon diameter in the presence of orientation dispersion [3] and undulation [4], grey matter imaging to discriminate cytoarchitectures [5], and muscle imaging to assess the degree of injury [6].

Microstructure imaging uses mathematical models to relate tissue features directly to the signal and thus supports estimates of specific tissue parameters from combinations of diffusion MRI measurements. The single diffusion encoding (SDE) sequence $[7,8]$ is the standard pulse sequence for acquiring diffusion MRI data. A collection of SDE measurements is sensitive to pore-size distribution in known isotropic pores or coherently oriented anisotropic pores. This has led to various imaging techniques using SDE to make estimates of axon density, diameter distribution $[9,10,11]$ and fibre dispersion [12] in white matter as well as tumour specific microstructural indices such as cell size and density, vascular volume fraction and cellularity [1]. However SDE sequences fail to discriminate between more complex systems, such as certain configurations of isotropic pores with a size distribution and randomly oriented anisotropic pores [13].

More sophisticated pulse sequences, in particular those that have varying gradient orientation within one measurement such as double diffusion encoding (DDE) sequences $[14,8]$ can remove some of this ambiguity [15]. As illustrated in Figure 1a), the DDE sequence concatenates two SDE sequences separated by a mixing time. Thus, it measures the correlation of water displacement at different times and/or in different directions. This can provide sensitivity to features less visible to SDE sequences, such as pore shape or exchange rate. In practice, protocols of DDE measurements cover different subsets of the full measurement space depending on what features they intend to investigate. For example, in the limit of short pulses, long diffusion time and short mixing time, pairs of diffusion gradients with the same amplitude and varying orientations have been used to estimate pore size [16, 17, 18, 19]. 
In the same limit, [20] showed that DDE sequences preserve the diffusion-diffraction patterns in the case of a distribution of sizes. In the long mixing time regime, pairs of diffusion gradients with the same orientation and varying amplitudes have been used to measure exchange $[21,22]$. If, on the other hand, the gradient amplitudes are fixed, the dependence of diffusion signal on the angle between the two gradients reflects pore eccentricity $[15,13]$. The diffusion signal from angular DDE measurements has been investigated in various studies that cover simulations [13, 23], phantom experiments $[24,17,25]$ as well as ex-vivo and in-vivo imaging [26, 27, 28]. Most of these studies assume macroscopically isotropic substrates or known fibre orientation. The residual ensemble anisotropy is modelled in [26] as a phase shift in the angular dependence of the signal, while more recent studies propose rotationally invariant metrics, such as the microscopic anisotropy $(M A)[29]$ index or fractional eccentricity $(F E)$ [30]. These metrics of microscopic anisotropy are obtained by contrasting DDE measurements with parallel and perpendicular gradients. A similar metric, microscopic fractional anisotropy $(\mu F A)[31]$, derived assuming time independent diffusivities, uses a combination of gradient waveforms which provide isotropic diffusion weighting. Other studies use DDE measurements for estimating intrinsic microstructural features. For instance, a model of identical pores featuring microscopic anisotropy [13] has been employed to show differences between isotropic substrates in the special case of spherical pores or randomly oriented cylindrical pores [25] and a more recent study has investigated the ability of SDE and DDE sequences to recover pore size [32] and length distributions [33] in cylindrical pores with known orientation.

In this paper we demonstrate in simulation the feasibility of using a model-based approach to provide quantitative microstructural features for a wide range of pores with various elongations, size distributions and orientation dispersions. First, we investigate the dependence of standard indices of microscopic anisotropy on substrate parameters, then we show that more specific parameters such as pore size and eccentricity can be estimated by fitting a microstructural model to the diffusion data. We test whether a simple model with identical pores can provide a good estimate of volume-weighted mean pore size and eccentricity and we investigate the effects of explicitly modelling pore size distribution. Additionally, we compare the ability of different protocols with SDE and DDE sequences to recover the ground truth parameters. We further hypothesize that such a model-based technique can be extended to analyse macroscopically anisotropic substrates as well as substrates with various parametric and non-parametric size distributions. 


\section{Methods}

\subsection{Data synthesis}

To synthesize diffusion data we use the MC simulator in Camino [34] using a mesh-based substrate, with closed pores represented as cuboids. The diffusion meshes have 1000 cuboids with two equal sides $\left(l_{x}=l_{y}\right)$ and a gamma distribution of sizes, as illustrated in Fig.1b in the case of a macroscopically isotropic substrate. The parameters of the model are the mean width of the cuboid $\bar{l}_{x}$, the ratio between the height and width $E=l_{z} / l_{x}$ (eccentricity), which is the same for all pores regardless of size, the gamma distribution shape parameter $a$ and the diffusivity constant $D$. We choose different geometric models to synthesize and fit the data in order to emphasize the robustness of this approach when the geometry is not a perfect match. Moreover, we prefer cuboids over ellipsoids due to the reduced computational complexity of the mesh. All MC simulations have 1000 time steps and 200000 walkers located inside the pores. Thus the synthesized data has intra-cuboidal signal only. Noise, with a Rician distribution and a signal-to-noise ratio (SNR) of 50, was added to the data to create 100 different data sets for each substrate.

\subsection{Signal model and fitting}

For fast signal computation, we expand the Gaussian Phase Distribution (GPD) approximation [35] for DDE sequences and a restriction model of finite cylinders with various sizes. Previous work [36] derived analytical expressions for DDE sequences and a similar restriction model keeping terms up to second order in $2 \pi q R$, where $q$ is the wavenumber and $R$ is the radius. Here we keep second order terms in the expansion of the signal logarithm (cumulant expansion) which has a wider range of applicability than the same order of the Taylor series [37]. The GPD approximation provides analytical expressions of the signal $S(\mathbf{u}, R, E)$ for a finite cylinder with orientation $\mathbf{u}$, radius $R$ and length $2 R E$. We use the same framework presented in [38] for oscillating gradients.

To calculate the signal for an ensemble of finite cylinders, we numerically integrate the signal over orientation and size distribution weighted by volume, to obtain the overall signal $S=\int_{\mathbf{u}} \int_{0}^{\infty} \mathcal{P}(R) \mathcal{F}(\mathbf{u}) S(\mathbf{u}, R, E) d \mathbf{u} R^{3} d R . \quad \mathcal{P}(R)$ is the probability distribution of radii, $\mathcal{F}(\mathbf{u})$ is the probability distribution of cylinder orientation and $E$ is fixed. The factor $R^{3}$ arises because the diffusion MRI signal from each pore depends on the amount of spins it contains and in our model the length of the cylinder is proportional to the radius. Assuming independence of parallel and perpendicular displacements [39], the signal for one cylinder is then 


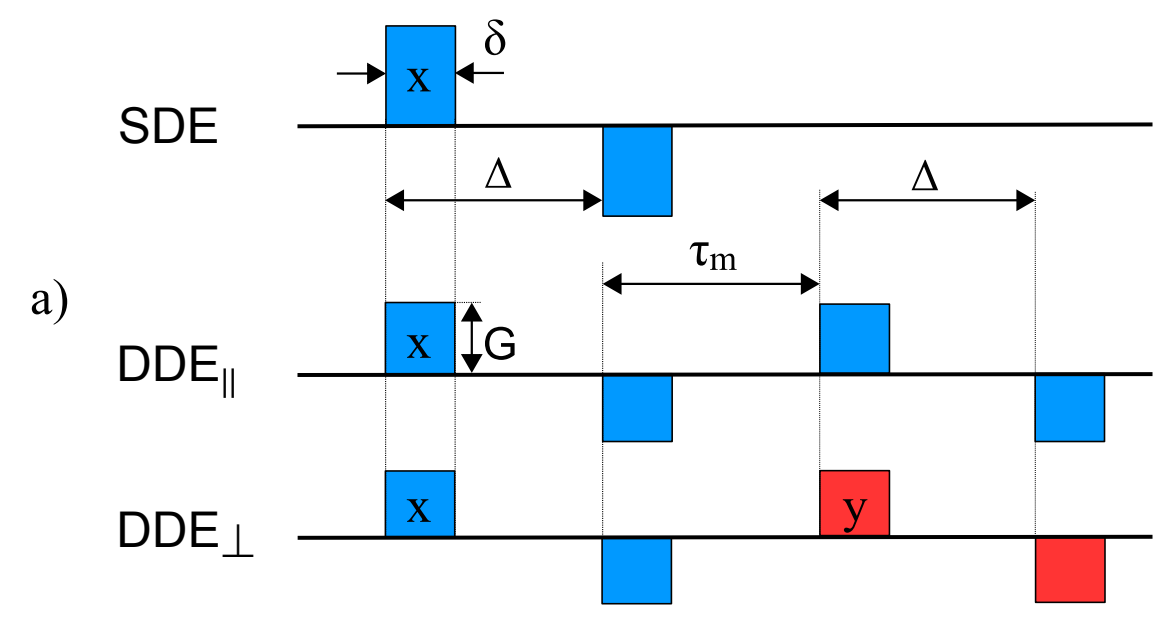

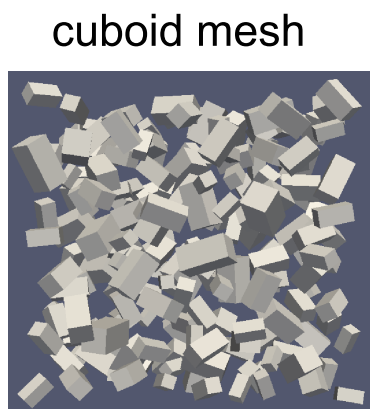

b)

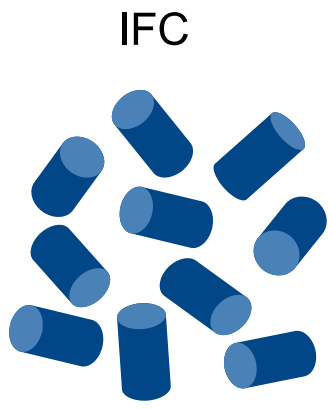

c)

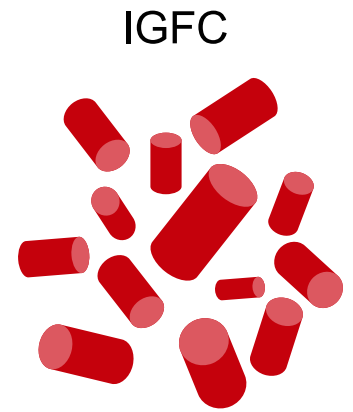

d)

Figure 1: a) Effective diffusion gradient waveforms (accounting for the effect of $180^{\circ}$ rf pulses) for SDE, DDE with parallel gradients and with perpendicular gradients. b) Isotropic mesh based diffusion substrate for MC simulations $\left(\overline{l_{x}}=6 \mu \mathrm{m}, E=2, a=2.5\right)$. c) Schematic representation of the IFC model. d) Schematic representation of the IGFC model

$$
\begin{aligned}
& S(\mathbf{u}, R, E)=S_{\perp}(\mathbf{u}, R, E) S_{\|}(\mathbf{u}, R, E) \text { with } \\
& \ln S_{\perp}(\mathbf{u}, R, E)= \frac{\gamma^{2}}{2} \sum_{n} B_{c y l, n} \int_{0}^{T E} d t_{1} \int_{0}^{T E} d t_{2} \exp \left(-\lambda_{c y l, n} D\left|t_{2}-t_{1}\right|\right) \\
& \mathbf{G}_{\perp}\left(t_{1}\right) \cdot \mathbf{G}_{\perp}\left(t_{2}\right) \\
& \ln S_{\|}(\mathbf{u}, R, E)= \frac{\gamma^{2}}{2} \sum_{n} B_{\text {plane }, n} \int_{0}^{T E} d t_{1} \int_{0}^{T E} d t_{2} \exp \left(-\lambda_{\text {plane }, n} D\left|t_{2}-t_{1}\right|\right) \\
& G_{\|}\left(t_{1}\right) G_{\|}\left(t_{2}\right)
\end{aligned}
$$


where $\gamma$ is the gyromagnetic ratio $B_{n}$ and $\lambda_{n}$ are geometry related factors for cylindrical and planar restriction [40] which depends on $R$ and $E, G_{\perp}$ and $G_{\|}$are the components of the diffusion gradient perpendicular and parallel, respectively, to the cylinder axis and $T E$ is the echo time. The full expressions of the signal are provided in the Supplementary Material.

We fit the models to data in Matlab using a two-step procedure: a grid search of predefined values, which gives a rough estimates of parameters, followed by a gradient descent which minimizes the difference between the data and the model given Rician noise. During all stages of fitting $D$ is fixed to its true value. For numerical stability, the lower limit of $a$ in the IGFC model is set to 1 and the upper limit to 10000 . All other parameters are estimated with no constraints. We also ensured that the parameter values used in the grid search do not overlap with simulation parameters. For the anisotropic substrates, the main orientation is estimated from a DTI data set, by fitting a linear diffusion tensor model, and fixed afterwards. This reduces the computational complexity of the gradient descent without compromising on accuracy.

\subsection{Simulation 1: Metrics of microscopic anisotropy}

Derivation of $\epsilon$ and $F E$

This experiment investigates the dependence of eccentricity $(\epsilon)$ and the normalized metric fractional eccentricity $(F E)[30]$ on substrate parameters. We use the expressions and the acquisition protocol derived in [30]. Thus, the measurements consist of 12 DDE sequences with parallel gradients and 60 DDE sequences with perpendicular gradients, with the directions defined in [30]. The two metrics of microscopic anisotropy, $\epsilon$ and $F E$, are derived based on the difference between the measurements with parallel and perpendicular gradients:

$$
\log \left(\frac{1}{12} \sum S_{\|}\right)-\log \left(\frac{1}{60} \sum S_{\perp}\right)=q^{4} \epsilon
$$

where $S_{\|}$is the signal for the DDE sequences with parallel gradients, $S_{\perp}$ is the signal for the DDE sequences with perpendicular gradients, $q=\gamma G \delta$ is the wavenumber, $\gamma$ the gyromagnetic ratio , $G$ the gradient strength, $\delta$ the pulse duration and $\epsilon$ depends on pore size and eccentricity. For spherical pores $\epsilon=0$. Fractional eccentricity normalizes $\epsilon$ with respect to size:

$$
F E=\sqrt{\frac{\epsilon}{\epsilon+3 \Delta^{2} \bar{D}^{2} / 5}}
$$

which varies between 0 (spherical pores) and 1 (elongated pores), where $\Delta$ is the diffusion time and $\bar{D}$ is the mean diffusivity calculated from the measurements with parallel gradients. 


\section{Diffusion substrates}

To analyse the dependence of $\epsilon$ and $F E$ on pore eccentricity we construct diffusion substrates as explained in section 2.1 with the following parameters: $\overline{l_{x}}=$ $\{4,8,12\} \mu \mathrm{m}, a=\{2.5,10, \infty\}(a \rightarrow \infty$ yields identical pores) and $E$ varying between 1 and 3 .

\section{Measurement protocol}

Further, we choose DDE sequences with parallel and perpendicular gradients with long diffusion and mixing times and pulse duration of $\delta=5 \mathrm{~ms}$. To have a similar diffusion attenuation for all substrates and to be in the same diffusion regime we keep constant $\gamma \delta G \overline{l_{x}}$ and $\Delta D /\left(\overline{l_{x}}\right)^{2}$, respectively, as explained in [41]. The gradient strength corresponding to the substrates with $\overline{l_{x}}=4 \mu \mathrm{m}$ is $G=300 \mathrm{mT} / \mathrm{m}$ and decreases accordingly for larger pores. This ensures the signal attenuation is above the noise floor for all substrates, with values between 0.9 and 0.2 . The diffusion and mixing times, which are $\tau_{m}=\Delta=100 \mathrm{~ms}$ for the substrates with $\overline{l_{x}}=4 \mu \mathrm{m}$, are increased for larger pores, yielding a constant ratio $\Delta D / \bar{l}_{x}^{2}=12.5$. Additionally, we investigate the effect of noise with three different SNR levels of 50, 100 and $\infty$. As $S_{\|}-S_{\perp} \geq 0$ [13], the negative values of $\epsilon$ occur solely due to noise and are set to 0 , in order to obtain real valued $F E$.

\subsection{Simulation 2: Macroscopically isotropic substrates}

\section{Diffusion substrates}

This simulation explores a model-based approach for estimating pore size and eccentricity in macroscopically isotropic substrates that have a distribution of pore sizes. To this end, we construct separate substrates for each combination with the following parameter values: $\overline{l_{x}}=\{2,4, \ldots 12\} \mu \mathrm{m}, E=\{1,1.5,2,2.5,3\}, a=\{2.5,10, \infty\}$ and $D=2 \cdot 10^{-9} \mathrm{~m}^{2} / \mathrm{s}$. The smaller pores have similar sizes to axons and dendrites in brain tissue [42, 43], while larger pores are closer to certain cancer cells, e.g. [44]. For intrinsic diffusivity we chose a value similar to the principal eigenvalue of the diffusion tensor measured at short diffusion time in the human brain [45].

\section{Signal model}

For the model-based estimation of pore size and eccentricity in a macroscopically isotropic substrate, we construct two signal models. The first one has identical pores and aims to test whether a simpler model can recover the average volume weighted size and eccentricity, in a similar way ActiveAx [10] yields an index of axon diameter. The second model explicitly accounts for pore size distribution. As we are investigating macroscopically isotropic substrates, the probability distribution of 
cylinder orientation is the 0 th order spherical harmonic $(\mathcal{F}(\mathbf{u})=1 / \sqrt{4 \pi})$. The two models are:

1. Isotropic finite cylinders (IFC) consists of randomly oriented identical finite cylinders and is illustrated in Fig.1c. This model has three parameters: cylinder radius $R$, ratio between cylinder length and diameter $E$ (eccentricity) and diffusivity constant $D$. In this case the probability distribution of radii is a delta function $\mathcal{P}(R)=\delta(R)$.

2. Isotropic gamma finite cylinders (IGFC) consists of randomly oriented finite cylinders with a volume weighted gamma distribution of radii, so explicitly accounts for a size distribution, as illustrated in Fig. 1d. This model has four parameters: $D$, mean radius $\bar{R}$, the gamma distribution shape parameter $a$, and eccentricity $E$, which is the same across all sizes. In this case the probability distribution of radii is $\mathcal{P}(R)=\frac{1}{C} \frac{R^{a-1}(a / \bar{R})^{a} \exp (-R a / \bar{R})}{\Gamma[a]}$, where $\Gamma(a)$ is the Gamma function and $C=\int_{0}^{\infty} \frac{R^{a-1}(a / \bar{R})^{a} \exp (-R a / \bar{R})}{\Gamma[a]} R^{3} d R$ is a normalisation constant which accounts for volume weighting. The variance of the distribution is $\operatorname{var}\{\mathcal{P}(R)\}=\bar{R}^{2} / a$.

\section{Measurement protocol}

For estimating microstructural parameters, we test four different measurement protocols, constructed from basic SDE and DDE sequences shown in Fig. 1a. We construct a rich protocol for each sequence type to ensure sensitivity across as wide a range of pore sizes as possible. As the substrates are macroscopically isotropic, the diffusion gradients are only in $\mathrm{x}$ direction (parallel) and y direction (perpendicular). To make the comparison as fair as possible, we choose sequence parameters that yield the same maximum diffusion weighting (b-value) and number of measurements in each protocol. The protocols are:

1. SDE protocol. This has the following parameters: pulse duration $\delta=\{5,10,, \ldots 25\}$ ms, gradient strength $G=\{25,50,75,100,300,500\} \sqrt{2} \mathrm{mT} / \mathrm{m}$ and time interval between the beginning of the first and second gradients $\Delta=\delta+\{5,10,20,30,40\}$ ms with two repetitions for each measurement. The gradient strength for SDE measurements is higher by a factor of $\sqrt{2}$ in order to have the same b-value as the other protocols.

2. DDE $_{\|}$protocol. This has DDE sequences with parallel gradients of equal amplitudes. The other parameters are: $\delta=\{5,10, \ldots 25\} \mathrm{ms}, \Delta=\delta+$ $\{5,10,20,30,40\} \mathrm{ms}, G=\{25,50,75,100,300,500\} \mathrm{mT} / \mathrm{m}$, and mixing time $t_{m}=\Delta$, with two repetitions for each measurement. 
3. $\mathbf{D D E}_{\perp}$ protocol. This has DDE sequences with perpendicular gradients of equal amplitudes. The rest of the parameters are the same as for $\mathbf{D D E} \mathbf{E}_{\|}$.

4. $\mathbf{D D E}_{\| \& \perp}$ protocol. This has DDE sequences with both parallel and perpendicular gradients for each combination of parameters in the $\mathbf{D D E}_{\|}$. Including only one repetition rather than two in the $\mathbf{S D E}, \mathbf{D D E}_{\|}$and $\mathbf{D D E}_{\perp}$ protocols ensures the same number of measurements in all protocols.

\section{Protocol comparison}

For a quantitative protocol comparison, we use an objective function derived from the Cramer-Rao Lower Bound (CRLB) to rank the four protocols. The CRLB provides a lower bound on the variance of the parameter estimates given a set of measurements, and often is closely correlated to the true variance. Thus, objective functions based on the CRLBs are a standard optimality criterion and have been previously used in diffusion MRI experiment design [46, 47]. To ensure similar scale, we use the sum of the normalized CRLBs to compare the four protocols:

$$
F=\sum_{i}^{P}\left(J^{-1}\right)_{i i} / p_{i}^{2}
$$

where $p_{i}$ are the model parameters with $i=1, \ldots P, J$ is the Fisher information matrix and $\left(J^{-1}\right)_{i i}$ is the CRLB for $p_{i}$.

\subsection{Simulation 3: Macroscopically anisotropic substrates}

\section{Diffusion substrates}

This simulation tests the hypothesis that a model-based approach can be extended to estimate pore size and eccentricity in the presence of macroscopic anisotropy. To this end, we construct diffusion substrates consisting of cuboids oriented according to a Watson distribution, as explained in section 2.1. The substrates have the largest size variance $(a=2.5)$ and all combinations of $\overline{l_{x}}$ and $E$ presented in Simulation 2, as well as various concentration parameters of the Watson distribution $\kappa=\{2,4,8,16,32\}$.

\section{Signal model}

For a macroscopically anisotropic substrate, in order to recover rotationally invariant indices of pore size and eccentricity, we explicitly model the orientation distribution of the pores as a Watson distribution, i.e. $\mathcal{F}(\mathbf{u})=W\left(\frac{1}{2}, \frac{3}{2}, \kappa\right)^{-1} e^{\kappa(\boldsymbol{\mu} \cdot \mathbf{u})^{2}}$, where $W$ is a confluent hypergeometric function, $\kappa$ is the concentration parameter and $\boldsymbol{\mu}$ is the main orientation. Thus, the new models are: 
1. Watson finite cylinders (WFC) has three additional parameters compared to the IFC model: concentration parameter of the Watson distribution $\kappa$ and the angles $\theta$ and $\phi$ in spherical coordinates describing the main direction $\boldsymbol{\mu}$.

2. Watson gamma finite cylinders (WGFC) has three additional parameters compared to the IGFC model: concentration parameter of the Watson distribution $\kappa$ and the angles $\theta$ and $\phi$ in spherical coordinates describing the main direction $\boldsymbol{\mu}$.

\section{Measurement protocol}

In this experiment we extend the measurement protocol to provide directional information. To measure the dominant orientation of pores, we use a DTI-like measurement set with 32 isotropically distributed directions and a b-value of $2000 \mathrm{~mm}^{2} / \mathrm{s}$ ( $\mathrm{G}=100 \mathrm{mT} / \mathrm{m}, \delta=10 \mathrm{~ms}$ and $\Delta=31 \mathrm{~ms}$ ). We chose a higher b value compared to the standard $\mathrm{b}=1000 \mathrm{~mm}^{2} / \mathrm{s}$ DTI measurements as we are probing restricted diffusion only. To measure pore size and eccentricity as well as orientation dispersion we use DDE sequences with 6 gradient orientations (xx,xy,xz,yy,yz,zz) for each combination of parameters in protocol $\mathbf{D D E}_{\|}$. This is the minimum number of gradient directions to ensure that parallel and perpendicular measurements along the three orthogonal axes are acquired. We refer to this protocol, which is designed to enable estimates of pore size and eccentricity in macroscopically anisotropic substrates with unknown orientation, as a rotationally invariant protocol $\mathbf{D D E}_{R I}$.

\subsection{Simulation 4: Varying size and orientation distribution}

In Simulation 2 and 3, both the diffusion substrates and the signal model use a Gamma distribution for pore sizes and a Watson distribution for pore orientation. In this section we relax these assumptions and test the applicability of a model-based approach when the simulated data and signal model have different distributions of pore size and orientation.

\section{Different size distributions}

Here we investigate the effects of fitting the $I G F C$ model to diffusion substrates consisting of elongated cuboids that have different size distributions. First we test a different parametric distribution, namely inverse Gaussian, then a realistic histological distribution taken from histology of SW-620 cell line of colorectal cancer [48]. In both cases we simulate the diffusion signal from the $\mathbf{D D E} \mathbf{E}_{\| \& \perp}$ protocol.

In the first simulation we analyse meshes consisting of randomly oriented cuboids with eccentricity $E=2$ and a size distribution given by an inverse Gaussian with mean size $\bar{l}_{x}=6 \mu \mathrm{m}$ and shape parameter $\lambda=\{10,50,100\} \mu \mathrm{m}$. As the shape parameter increases, the inverse Gaussian tends towards a normal distribution. 
In the second simulation we construct a cuboid mesh with the discrete size distribution from histology of SW-620 colorectal cancer cells [48], which has a mean value $\overline{l_{x}}=10.8 \mu \mathrm{m}$ and we assume a smaller eccentricity $E=1.5$.

\section{Different orientation distributions}

In this experiment we relax the assumption that the diffusion substrate and signal model have the same orientation distribution. Thus, we analyse the effect of fitting the Watson distribution to diffusion signal originating from substrates with a combination of coherently oriented cuboids occupying a volume fraction $f=\{0.25,0.5,0.75\}$ and randomly oriented cuboids with volume fraction $1-f$. The cuboids have a gamma distribution of sizes with shape parameter $a=2.5$ and mean value $\overline{l_{x}}=6 \mu \mathrm{m}$. To maximize the effect of orientation distribution we choose the largest eccentricity used in previous simulations $E=3$. The diffusion signal is synthesized for the $\mathbf{D D E}_{\mathbf{R I}}$ protocol and we fit the WGFC model.

\section{Results}

\subsection{Simulation 1: Metrics of microscopic anisotropy}

In the first simulation we investigate the dependence of $F E$ and $\epsilon$ from Eq. 3 on pore elongation for various substrates and different noise levels $\operatorname{SNR}=\{50,100, \infty\}$. Figure 2a) plots $F E$ against cuboid eccentricity in the range $E \in[1,3]$ for substrates of various mean sizes $\bar{l}_{x}=\{4,8,12\} \mu \mathrm{m}$ and size distribution $a=\{2.5,10, \infty\}$. Figure $2 \mathrm{~b})$ shows a similar dependence for $\epsilon$ normalized with the pore size $\bar{l}_{x}$. The plots present the median values over 100 noise trials with SNR $=50$ (left), 100 (middle) and $\infty$ (right). Figure 2c) illustrates the $F E$ values pooled from substrates with different $\bar{l}_{x}$ and $a$ for different eccentricities $E=\{1,1.5,2,2.5,3\}$. The simulations show that $F E$ is very noisy and the values are overestimated for pores with low eccentricity, where the difference between the DDE measurements with parallel and perpendicular gradients is smaller than the standard deviation of the noise. The metric $\epsilon$, which is a direct measure of the signal difference, is less affected by noise, however it depends on both pore size and eccentricity.

In this experiment $F E$ and $\epsilon$ are computed assuming the long diffusion and mixing time limit for all substrates. If we keep the diffusion time the same for all substrates $\left(\tau_{m}=\Delta=100 \mathrm{~ms}\right)$, both $F E$ and $\epsilon$ are underestimated for the larger pores $\left(\bar{l}_{x}=12 \mu \mathrm{m}\right)$ (data not shown), which shows the importance of having the sequence parameters in the correct diffusion regime. 


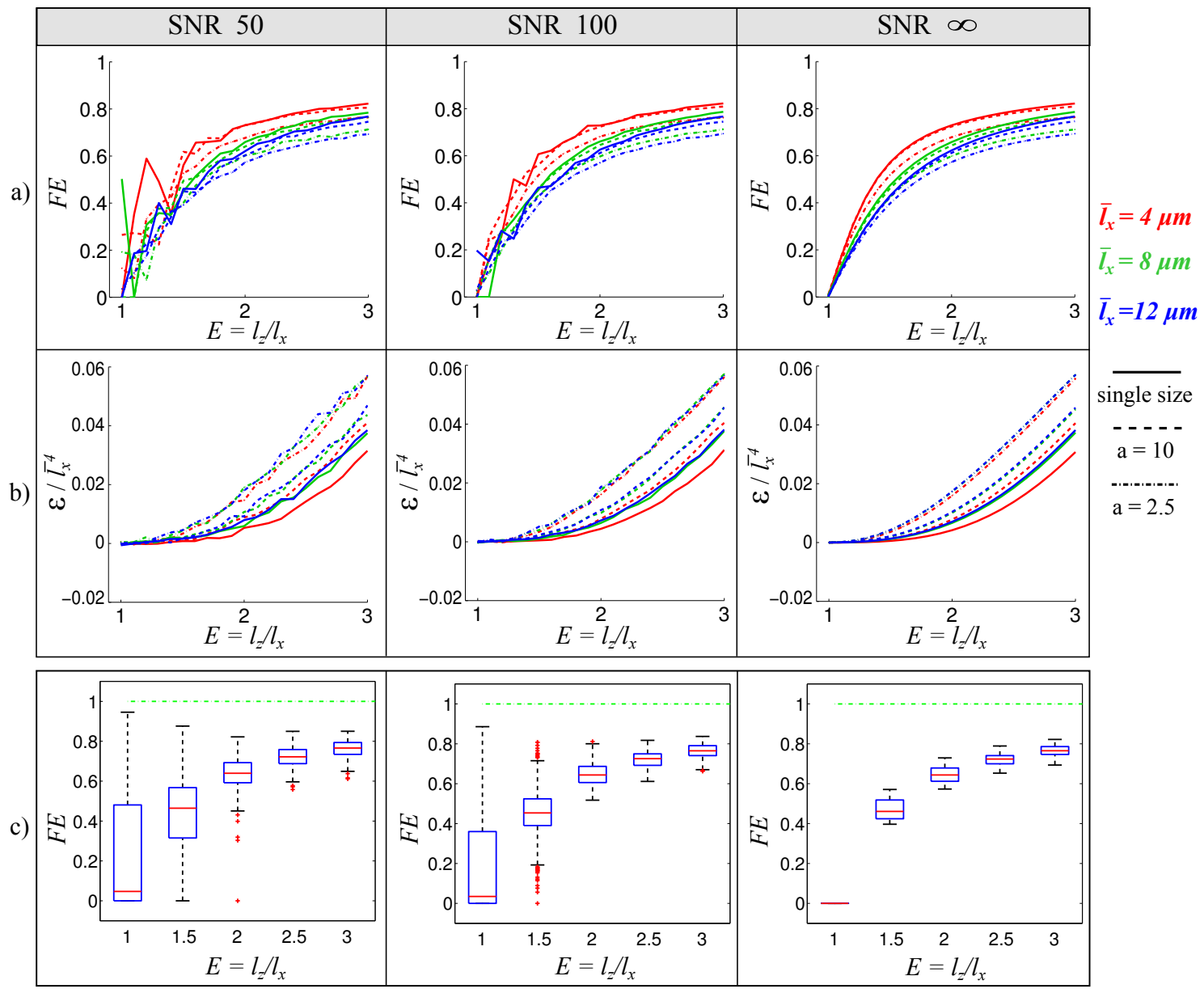

Figure 2: Dependence of a) $F E$ and b) $\epsilon$ on pore eccentricity. The plot illustrates the median values over 100 instances of noise with $\operatorname{SNR}=\{50,100$ and $\infty\}$. Different colours represent different sizes and different line styles represent different shapes of the gamma distribution. c) Box plots of $F E$ values for $E=\{1,1.5,2,2.5,3\}$ when the data is pooled from substrates with different mean sizes and shape parameters of the gamma distribution. The box plots present the median, 25th percentile (lower quartile) and 75th percentile (upper quartile) as well as data points that extend up to $1.5 \times$ inter-quartile range.

\subsection{Simulation 2: Macroscopically isotropic substrates}

This section focuses on model-based parameter estimation in macroscopically isotropic substrates. First we investigate the ability of a signal model with identical 
pores (IFC) to recover ground truth microstructural features in the case of substrates with a single pore size or a distribution of sizes. Then we analyse a signal model which directly accounts for the size distribution (IGFC). The last analysis of this section compares the sensitivity of the four protocols to recover the model parameters.

IFC model: Figure 3a) illustrates the relative error of the parameter estimates $\left(\Delta R=\left(R_{\text {est }}-R_{\text {g.t. }}\right) / R_{\text {g.t. }}\right)$ and $\left.\Delta E=\left(E_{\text {est }}-E_{\text {g.t. }}\right) / E_{\text {g.t. }}\right)$ from the IFC model for different ground truth values used in the $\mathrm{MC}$ simulation. When computing the relative errors, the ground truth parameter values are adapted to account for the difference in geometry between cuboids (MC simulation) and finite cylinders (signal model) by matching cylinder radius to give the same pore volume. Thus, for a cuboid with width $l_{x}$ and $E=l_{z} / l_{x}$, the corresponding radius of a finite cylinder is $l_{x} / \sqrt{\pi}$ and eccentricity is $E \sqrt{\pi} / 2$. The results are shown for SDE and $\mathrm{DDE}_{\| \& \perp}$ protocols. The relative errors are separated according to the ground truth values of the parameters, with radius estimates in the middle column and eccentricity estimates in the right column.

The relative errors of the IFC model parameter estimates ( $R$ and $E$ ) are reported in Table 1a) and 1b). As the values are not normally distributed, we report nonparametric statistics to control for outliers. The median, lower quartile and upper quartile of the relative errors are presented separately for each measurement protocol and size distribution. The results show that, if the diffusion substrate consists of identical pores, then a simple model of microscopic anisotropy, such as IFC, can be used to measure average size and eccentricity using any of the four protocols. Most outliers illustrated in Figure 3a) occur for the substrates with $l_{x}=2 \mu \mathrm{m}$, because the size reaches the lower bound on the range of sizes to which the measurements are sensitive with $G_{\max }=500 \sqrt{2} \mathrm{mT} / \mathrm{m}$, as described in [49]. A key observation is that, for these simple substrates, the estimates based on SDE sequences have similar accuracy to those from DDE sequences, when a suitable model has been assumed. This happens because, for the subset of identical randomly oriented pores, the elongation contributes to the apparent size distribution in any given direction, which can be estimated using SDE measurements. Nevertheless, DDE acquisitions can provide information regarding the presence of microscopic anisotropy with no a-priori assumption.

Figure $3 \mathrm{~b}$ ) illustrates the relative errors of the parameter estimates from the IFC model for a substrate with a gamma distribution of sizes, with shape parameter $a=2.5$. The results are presented for $\mathrm{SDE}$ and $\mathrm{DDE}_{\| \& \perp}$ sequences. In this case, when computing the relative errors, the ground truth parameter values are adapted to account both for the geometric correction explained above, as well as for volume weighting the size distribution, as discussed in section 2.2. The figure shows that, in 
a)

\begin{tabular}{|c|c|c|c|}
\hline & \multicolumn{3}{|c|}{ IFC - relative error of estimated radius $R: M d\left(q_{1}, q_{3}\right)$} \\
\hline & $a \rightarrow \infty$ & $a=10$ & $a=2.5$ \\
\hline SDE & $3.6(1.6,6.3) \%$ & $-9.6(-20,-2.9) \%$ & $-20(-32,-13) \%$ \\
\hline $\mathrm{DDE}_{\|}$ & $2.1(0.6,4.0) \%$ & $-12(-22,-5.7) \%$ & $-22(-33,-16) \%$ \\
\hline $\mathrm{DDE}_{\perp}$ & $0.4(-3.1,3.9) \%$ & $-18(-52,-11) \%$ & $-29(-68,-20) \%$ \\
\hline $\mathrm{DDE}_{\| \& \perp}$ & $1.6(-0.5,3.1) \%$ & $-12(-20,-5.8) \%$ & $-20(-31,-14) \%$ \\
\hline
\end{tabular}

b)

\begin{tabular}{|c|c|c|c|}
\cline { 2 - 4 } \multicolumn{1}{c|}{} & \multicolumn{3}{c|}{ IFC - relative error of estimated eccentricity $E: M d\left(q_{1}, q_{3}\right)$} \\
\cline { 2 - 4 } \multicolumn{1}{c|}{} & $a \rightarrow \infty$ & $a=10$ & $a=2.5$ \\
\hline $\mathrm{SDE}$ & $-0.8(-5.5,1.4) \%$ & $28(16,72) \%$ & $103(58,192) \%$ \\
\hline $\mathrm{DDE}_{\|}$ & $-1.7(-4.6,0.1) \%$ & $27(11,77) \%$ & $87(38,186) \%$ \\
\hline $\mathrm{DDE}_{\perp}$ & $0.9(-3.8,7.5) \%$ & $35(18,140) \%$ & $92(47,329) \%$ \\
\hline $\mathrm{DDE}_{\| \& \perp}$ & $-0.2(-3.2,1.4) \%$ & $20(8.5,58) \%$ & $50(25,105) \%$ \\
\hline
\end{tabular}

Table 1: Median $(M d)$, lower quartile $\left(q_{1}\right)$ and upper quartile $\left(q_{2}\right)$ of the relative error of estimated parameters from the IFC model: a) radius and b) eccentricity. For each measurement protocol and ground truth shape parameter $a$, the data is pooled from substrates with different mean radii and eccentricities. 
the presence of a distribution of sizes, the IFC model does not give consistent size and eccentricity estimates from either protocol, nevertheless, the $\mathrm{DDE}_{\| \& \perp}$ protocol yields the smallest relative errors compared to the other sequences, while the eccentricity estimates based on SDE measurements appear more biased. The volume weighted average radius is underestimated and the pore eccentricity is overestimated for all diffusion protocols for both $a=10$ and $a=2.5$ as detailed in Table 1a) and 1b). The eccentricity estimates have larger errors and more outliers, which mostly occur for pores with small size $\left(l_{x}=2 \mu \mathrm{m}\right)$. The bias in parameter estimation increases with the variance of the size distribution. This effect is especially pronounced for pores with low eccentricity, when the IFC model explains the size variation as coming from pores with increased eccentricity.

IGFC model: Figure 4 presents the relative errors of the $\bar{R}$ and $E$ estimates from fitting the IGFC model to data synthesized from gamma distributed pores with shape parameter $a=2.5$ (the shape parameter that yields the largest variance considered in this study). The results are presented for all four protocols and are separated according to the ground truth values of the parameters. When computing the relative errors, the ground truth parameter values are adapted to account for the geometric correction and the volume weighting is incorporated in IGFC model. In comparison to the IFC model, explicitly accounting for size distribution improves the parameter estimates in more complex substrates from all measurement protocols. Fitted parameters from all protocols are close to the ground truth values used in the MC meshes with median relative errors less than 10\% for radius and less than $30 \%$ for eccentricity. Outliers occur for either very small pores, close to the lower bound of sensitivity [49], or for very large pores, when the longest diffusion time is too short to observe restriction. $\mathrm{DDE}_{\| \& \perp}$ sequences yield the smallest errors for both radius and eccentricity estimates. However, when an appropriate model is assumed, as it is the case here, all the protocols, including SDE, show reasonable sensitivity and enable estimation of all the parameters of the system.

Although not shown in Figure 4, the shape parameter of the gamma distribution was fitted as well. As the variance of the gamma distribution is inversely proportional to the shape parameter $\left(\operatorname{var}\{\mathcal{P}(R)\}=\bar{R}^{2} / a\right.$, see section 2.2), quantifying the error of the variance is more meaningful than quantifying the error of the shape parameter. Thus, the relative errors of mean radius, eccentricity and variance are reported in Table 2a), 2b) and 2c) for all substrates and measurement protocols. The SDE protocol yields the highest relative errors in estimating the size distribution variance which is also reflected by poorer estimates of $\bar{R}$ and $E$. The relative errors of the estimated variance are slightly higher, as it depends on both mean radius and shape parameter estimates. 


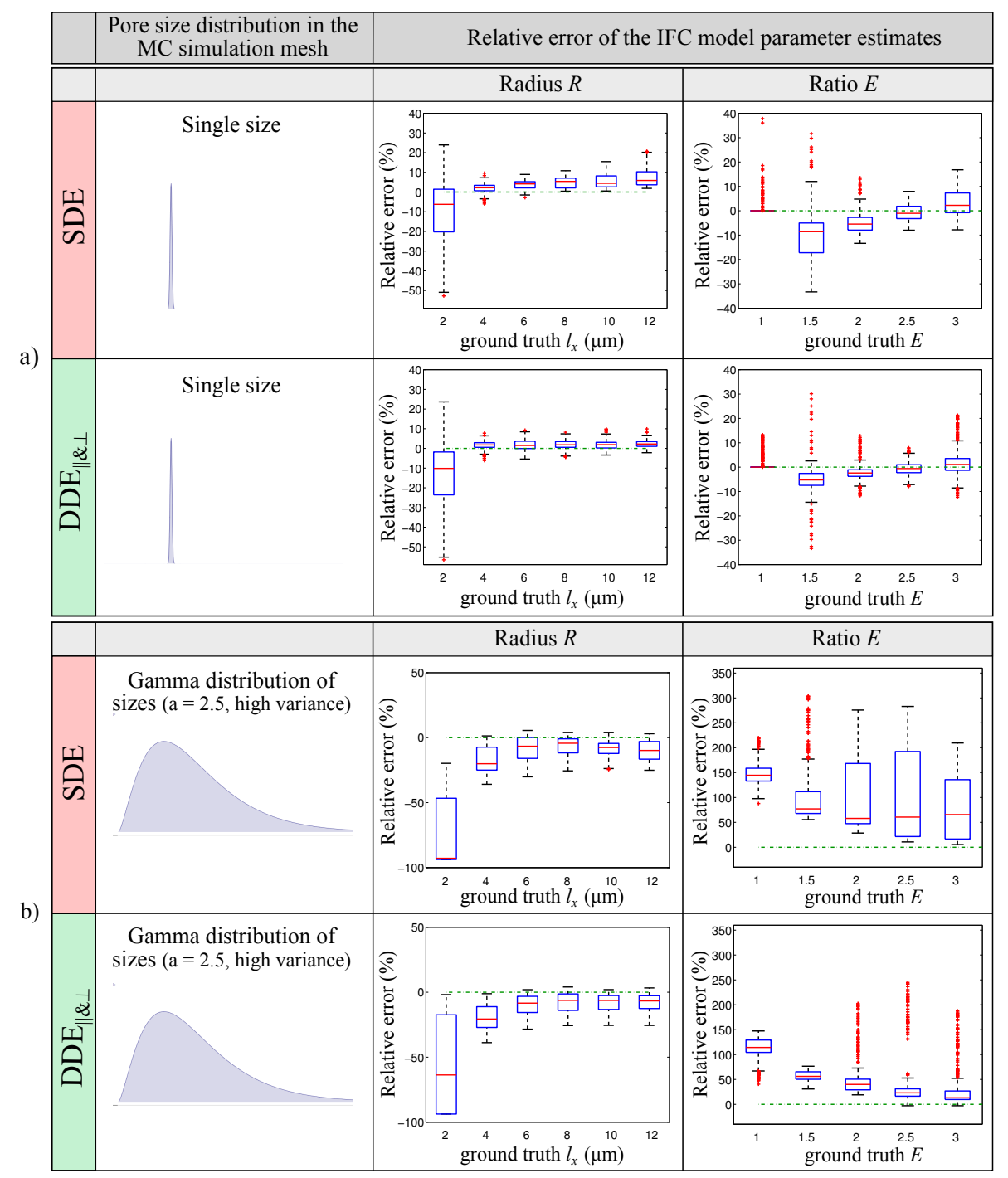

Figure 3: Relative errors of estimated parameters of the IFC model for different ground truth values in MC simulations, for a) a substrate with identical pores and b) a gamma distribution of sizes. The data is pooled across all substrates with a given size (middle column) or eccentricity (right column) and 100 noise trials. The box plots present the median, 25th percentile (lower quartile) and 75th percentile (upper quartile) as well as data points that extent up to $1.5 \times$ inter-quartile range.

Protocol comparison: For a more quantitative protocol comparison, we use 


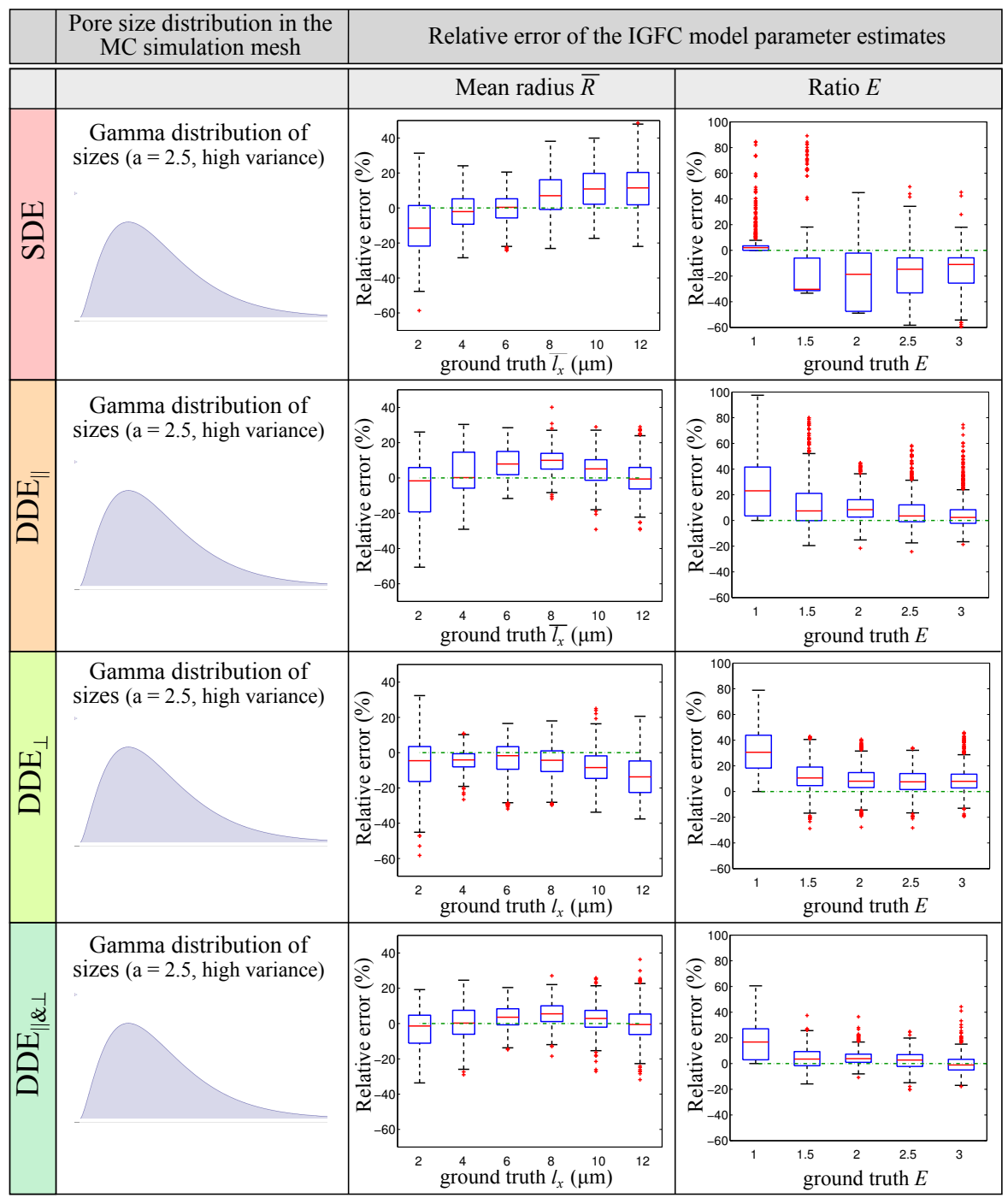

Figure 4: Relative errors of estimated parameters $(\bar{R}$ and $E)$ of the IGFC model for different ground truth values in $\mathrm{MC}$ simulations, for substrates with a gamma distribution of sizes $(a=2.5)$. The data is pooled across all substrates with a given size (middle column) or eccentricity (right column) and 100 noise trials.

the objective function $F$, defined in equation 4 as the sum of the normalized CRLBs of the model parameters. The lower the objective function, the smaller the expected variance of the estimated parameters. For the ICF model, Figure 5a) illustrates $F$ 
a)

\begin{tabular}{|c|c|c|c|}
\cline { 2 - 4 } \multicolumn{1}{c|}{} & \multicolumn{3}{c|}{ IGFC - relative error of estimated mean radius $\bar{R}: M d\left(q_{1}, q_{3}\right)$} \\
\cline { 2 - 4 } & & & \\
& & & \\
& & & \\
& & & \\
\hline $\mathrm{SDE}$ & $2.8(0.5,6.6) \%$ & $4.0(-0.3,7.5) \%$ & \\
\hline $\mathrm{DDE}_{\|}$ & $2.0(0.5,4.0) \%$ & $3.0(-0.4,5.6) \%$ & $2.8(-5.9,12) \%$ \\
\hline $\mathrm{DDE}_{\perp}$ & $0.4(-4.6,4.1) \%$ & $-2.1(-6.7,1.4) \%$ & $-5.6(-13,0.8) \%$ \\
\hline $\mathrm{DDE}_{\| \& \perp}$ & $1.4(-1.3,2.9) \%$ & $1.8(-1.0,4.1) \%$ & $1.9(-3.7,7.4) \%$ \\
\hline
\end{tabular}

b)

\begin{tabular}{|c|c|c|c|}
\cline { 2 - 4 } \multicolumn{1}{c|}{} & \multicolumn{3}{c|}{ IGFC - relative error of estimated eccentricity $E: M d\left(q_{1}, q_{3}\right)$} \\
\cline { 2 - 4 } \multicolumn{1}{c|}{} & $a \rightarrow \infty$ & $a=10$ & $a=2.5$ \\
\hline $\mathrm{SDE}$ & $-4.4(-14,0.1) \%$ & $-6.7(-20,0.1) \%$ & $-9.2(-31,0.1) \%$ \\
\hline $\mathrm{DDE}_{\|}$ & $-1.8(-4.9,0.1) \%$ & $4.6(0.1,12) \%$ & $7.4(0.1,23) \%$ \\
\hline $\mathrm{DDE}_{\perp}$ & $0.6(-4.3,7.1) \%$ & $8.5(2.3,17) \%$ & $11(4.2,23) \%$ \\
\hline $\mathrm{DDE}_{\| \& \perp}$ & $-0.2(-3.2,1.4) \%$ & $1.9(-1.0,6.7) \%$ & $1.5(-5.6,7) \%$ \\
\hline
\end{tabular}

c)

\begin{tabular}{|c|c|c|c|}
\cline { 2 - 4 } \multicolumn{1}{c|}{} & \multicolumn{3}{c|}{ IGFC - relative error of estimated variance $M d\left(q_{1}, q_{3}\right)$} \\
\cline { 2 - 4 } \multicolumn{1}{c|}{} & $a \rightarrow \infty$ & $a=10$ & $a=2.5$ \\
\hline $\mathrm{SDE}$ & $119(5.8,373) \%$ & $38(-8,87) \%$ & $38(10,61) \%$ \\
\hline $\mathrm{DDE}_{\|}$ & $4.8(2.1,10) \%$ & $-32(-52,-12) \%$ & $-19(-32,-2) \%$ \\
\hline $\mathrm{DDE}_{\perp}$ & $5.0(-1.9,39) \%$ & $-19(-31,-2.6) \%$ & $-8.5(-19,4.4) \%$ \\
\hline $\mathrm{DDE}_{\| \& \perp}$ & $4.4(0.8,9.5) \%$ & $-16(-29,-0.5) \%$ & $-8.6(-18,3.4) \%$ \\
\hline
\end{tabular}

Table 2: Median $(M d)$, lower quartile $\left(q_{1}\right)$ and upper quartile $\left(q_{2}\right)$ for the relative errors of the estimated parameters from the IGFC model: a) radius and b) eccentricity and c) variance. For each measurement protocol and ground truth shape parameter $a$, the data is pooled from substrates with different mean radii and eccentricities. For the substrates with identical pores, the upper limit of $a(a=10000)$ from the signal model was used to compute the variance.

for the four protocols as a function of eccentricity for substrates with identical pores $(a \rightarrow \infty)$ and two different radii $R=2$ and $4 \mu \mathrm{m}$. The plots show that $\mathrm{DDE}_{\perp}$ protocol has the highest $F$ (i.e. lowest sensitivity), while the other protocols have similar performance. The same trend appears for the other substrates. This result reflects with the data presented in Table 1 for $a \rightarrow \infty$, where $\mathrm{DDE}_{\perp}$ has the largest interquartile range for both radius and eccentricity estimates. Figure $5 b$ ) illustrates 
the objective function values for the IGFC model for substrates with $\bar{R}=2$ and $4 \mu \mathrm{m}$ and $a=2.5$. In this case the $\mathrm{DDE}_{\| \& \perp}$ and $\mathrm{DDE}_{\perp}$ protocols perform better than the protocols with one single gradient orientation. Table 2 supports this finding where, for substrates with $a=2.5$, the last two protocols have smaller interquartile ranges.

a)
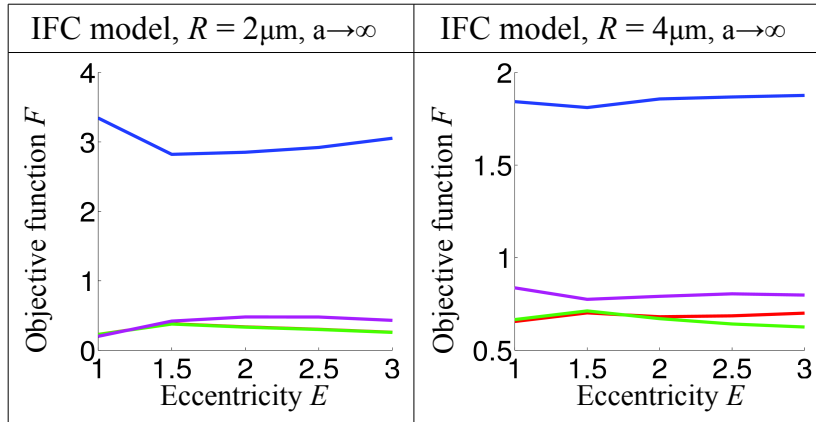

SDE

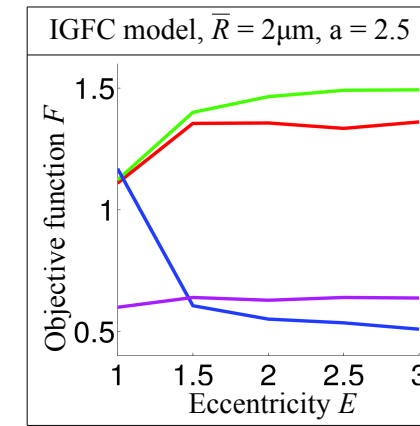

IGFC model, $\bar{R}=4 \mu \mathrm{m}, \mathrm{a}=2.5$

$\mathrm{DDE} \|$

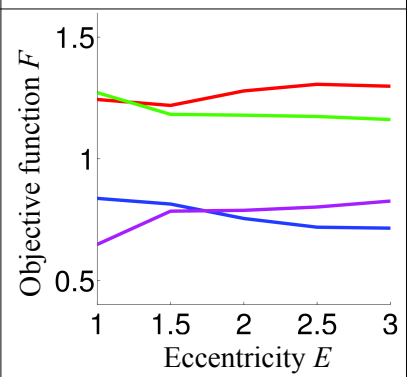

$\mathrm{DDE}_{\perp}$

$\mathrm{DDE} \| \& \perp$

Figure 5: Dependence of the objective function $F$ on pore eccentricity for the four protocols for a) the IFC model and b) the IGFC model.

\subsection{Simulation 3: Macroscopically anisotropic substrates}

This section presents the results for macroscopically anisotropic substrates. Having a rotationally invariant acquisition protocol and explicitly incorporating directional information in the tissue models allows for estimating size and eccentricity in the presence of macroscopic anisotropy. Thus we investigate the ability of signal models with a Watson orientation distribution to recover ground truth microstructural features.

As in the case of isotropically oriented pores, the WFC model with identical pores underestimates the radius and overestimates the eccentricity, especially in the case of pores with low eccentricity (data not shown). After accounting for the size 


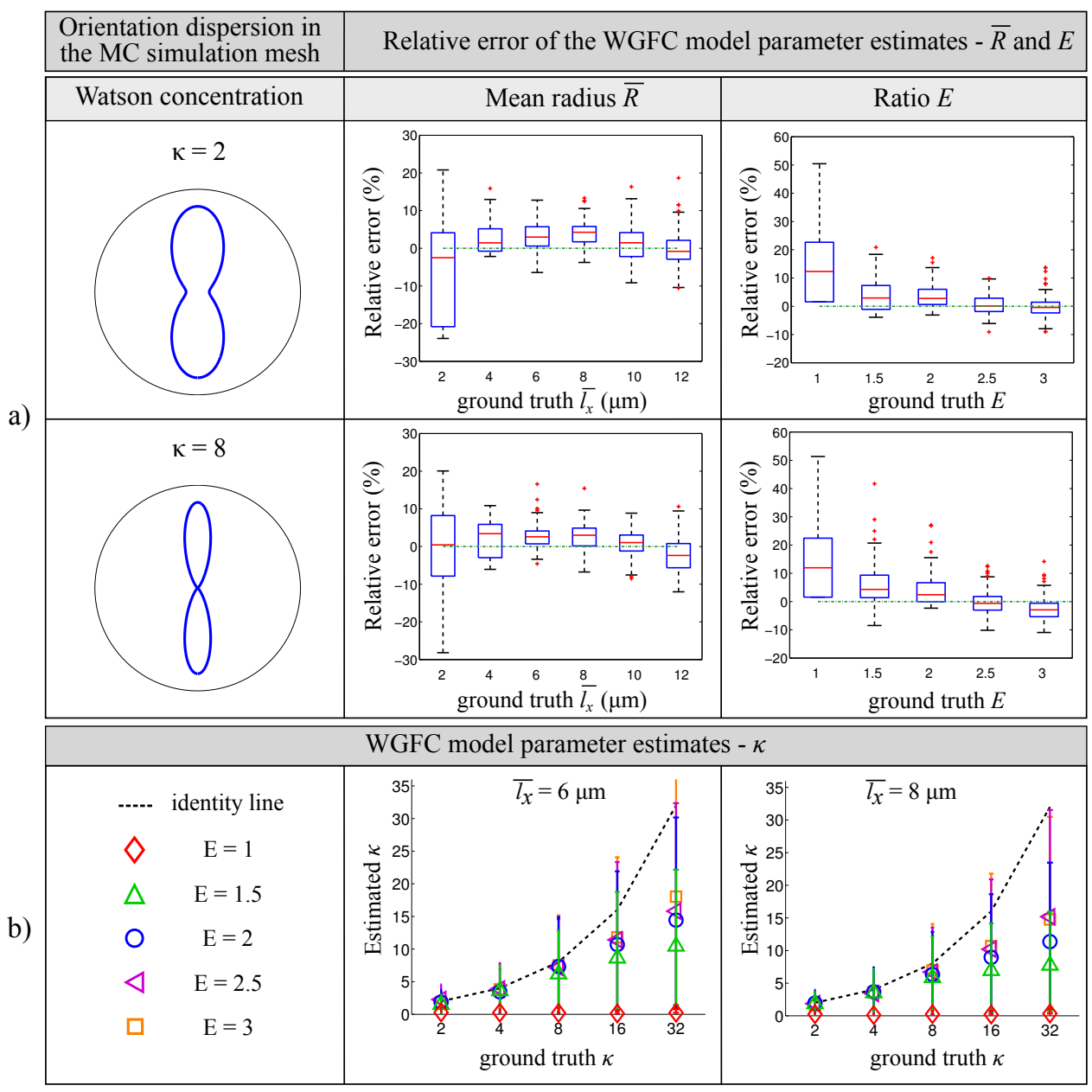

Figure 6: a) Relative error of the estimated size and eccentricity parameters from fitting the WGFC model a function of ground truth values in MC simulations, for a substrate with a gamma distribution of sizes $(a=2.5)$ and two different concentration parameters of the orientation distribution $(\kappa=2$ and 8$)$. The data is pooled across all substrates with a given size (middle column) or eccentricity (right column) and 100 noise trials. b) Estimated concentration parameter $\kappa$ of the WGFC model as a function of ground truth values used in MC simulations, for substrates with gamma distributed sizes $\left(\overline{l_{x}}=\{6,8\} \mu \mathrm{m}\right.$, $a=2.5)$ and various eccentricities. The values were computed as the mean estimates over 100 noise trials.

distribution of the pores, the WGFC model accurately recovers the ground truth parameters for mean radius and eccentricity. Figure 6a) illustrates the estimates of 
radius and eccentricity given by the WGFC model for substrates with two different orientation distributions $(\kappa=\{2,8\})$ and pores of various sizes and elongations. The shape parameter of the gamma distribution is $a=2.5$ for all substrates (largest variance considered in this study). The results show accurate estimates for mean radius and eccentricity for all $\kappa$ values. The median relative error of the estimated shape parameter $a$ is $4.8 \%$, with lower and upper quartiles of $-7.1 \%$ and $16 \%$, respectively. As for the isotropic substrates, larger errors occur for very small pores, close to the lower bound of sensitivity of the maximum gradient strength in this study.

Figure 6b) illustrates the estimates of the concentration parameter of the Watson distribution. For pores with low eccentricity $(E=1,1.5)$ the estimates of kappa are not accurate, as the choice of $\kappa$ becomes increasingly redundant as the pores approach isotropy. For elongated pores the estimated values of $\kappa$ are closer to the ground truth values from the MC simulations. As noted in [50], high $\kappa$ is hard to estimate precisely as the numerical value has an exponentially decreasing effect as $\kappa \rightarrow \infty$.

\subsection{Simulation 4: Varying size and orientation distribution}

Different size distributions

This section studies the accuracy of parameter estimates when the IGFC model is fitted to diffusion measurements from substrates with a different size distribution. We investigate both parametric and non-parametric distributions, as described in section 2.6 .

First we analyse substrates consisting of randomly oriented cuboids with eccentricity $E=2$ and an inverse Gaussian size distribution with mean value $\overline{l_{x}}=6 \mu \mathrm{m}$ and shape parameter $\lambda=\{10,50,100\} \mu \mathrm{m}$. The variance of the inverse Gaussian is $\bar{l}_{x}^{3} / \lambda$, and the distribution tends towards a normal distribution as the shape parameter increases. Fitting the $I G F C$ model to the diffusion data synthesized from the three meshes provides accurate estimates of mean radius and eccentricity, while the relative errors of the variance are larger. The size distribution of the cuboids as well as the relative errors of the parameter estimates are shown in Table 3a).

In the second simulation we evaluate the accuracy of the parameter estimates in a histological size distribution, as described in section 2.6. The average relative errors of the estimated IGFC model parameters over 10 noise trials with $\mathrm{SNR}=50$ are: $\overline{\Delta \bar{R}}=5.3 \pm 2.1 \%$ for the mean radius, $\overline{\Delta E}=1.1 \pm 3.8 \%$ for the eccentricity and $\overline{\Delta \operatorname{Var}}=-22 \pm 54 \%$ for the variance.

The results show that fitting the IGFC model to diffusion substrates with a different size distribution provides accurate estimates of average size and eccentricity, 


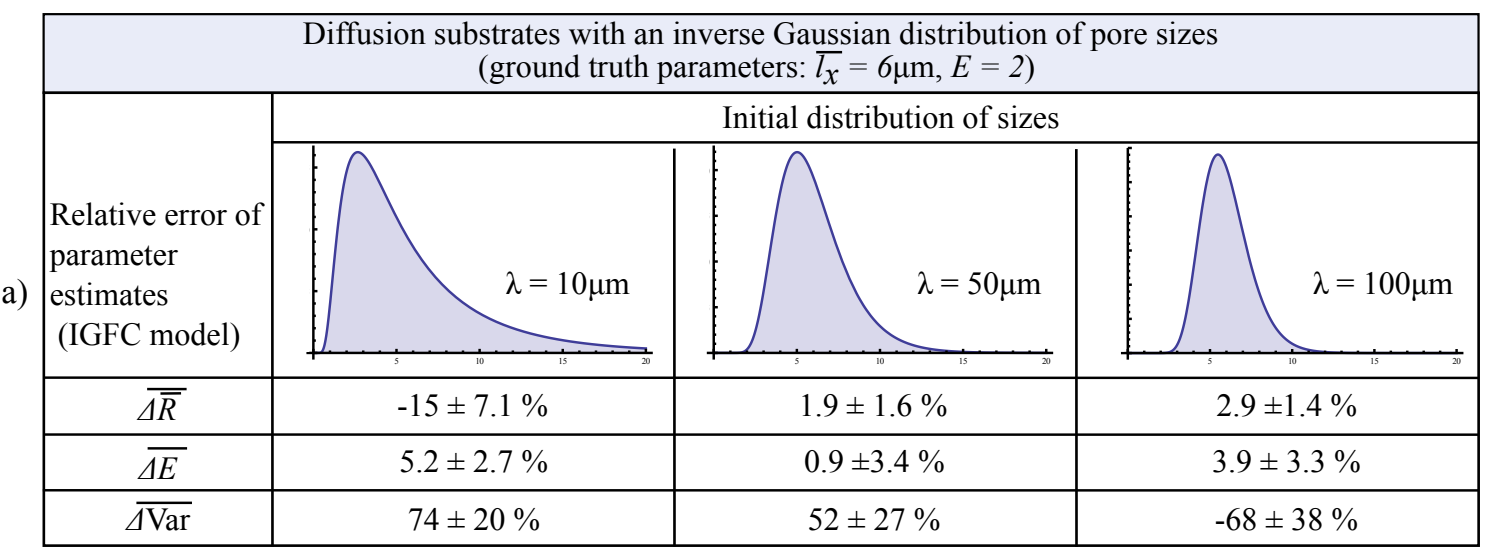

\begin{tabular}{|c|c|c|c|}
\hline \multicolumn{4}{|c|}{$\begin{array}{c}\text { Diffusion substrates with different orientation distributions } \\
\text { (ground truth parameters: } l_{x}=6 \mu \mathrm{m}, E=3, a=2.5 \text { ) }\end{array}$} \\
\hline \multirow{3}{*}{$\begin{array}{l}\text { Relative error of } \\
\text { parameter } \\
\text { estimates } \\
\text { bG }\end{array}$} & \multicolumn{3}{|c|}{ Volume fraction of coherently oriented pores $\mathrm{f}$} \\
\cline { 2 - 4 } & $\mathrm{f}=25 \%$ & $\mathrm{f}=50 \%$ & $\mathrm{f}=75 \%$ \\
\hline$\overline{\Delta \bar{R}}$ & $-3.3 \pm 3.8 \%$ & $-9.4 \pm 2.6 \%$ & $-12 \pm 6.8 \%$ \\
\hline$\overline{\Delta E}$ & $1.3 \pm 1.5 \%$ & $1.6 \pm 1.0 \%$ & $4.9 \pm 1.6 \%$ \\
\hline$\overline{\Delta \mathrm{Var}}$ & $-5.3 \pm 9.1 \%$ & $-4.3 \pm 7.5 \%$ & $12 \pm 17 \%$ \\
\hline
\end{tabular}

Table 3: a) Average relative error of estimated mean radius and eccentricity provided by the IGFC model when fitted to pores that have an inverse Gaussian distribution of sizes. b) Average relative error of estimated mean radius, eccentricity and shape parameter of the gamma distribution provided by the WGFC model when fitted to substrates with different volume fractions of coherently oriented pores. The mean value and standard deviation are computed over 10 noise trails with $S N R=50$.

for both parametric and non-parametric distributions. The estimated variance has the largest relative errors, which are further increased compared to the values in Simulation 2 and 3 due to the mismatch between the distributions.

\section{Different orientation distributions}

This simulation tests the ability of the WGFC model to estimate pore size and eccentricity in substrates that have a different orientation distribution. Thus we analysed substrates that have different volume fractions of coherently oriented and randomly oriented pores. The results presented in Table 3 show a good agreement 
between the estimated parameters of the WGFC model (mean radius, eccentricity and variance of the size distribution) and the ground truth values from the cuboid meshes. The estimated concentration parameter of the Watson distribution $\kappa$ increases $(\bar{\kappa}=1.5,2.7,4.9)$ with the volume fraction of the coherently oriented pores, reflecting the more anisotropic orientation distribution. These results illustrate that the WGFC model yields accurate estimates of pore size and eccentricity, even for a different orientation distribution of pores in the underlying substrate.

\section{Discussion}

This work is a proof of concept showing that microstructural parameters such as pore size and eccentricity can be estimated from diffusion MRI data using a geometric model of restriction even in the presence of macroscopic anisotropy and a distribution of pore sizes.

In Simulation 1 we investigated model-free metrics of microscopic anisotropy, specifically $F E$ and $\epsilon$ which are computed from the difference of DDE measurements with parallel and perpendicular gradients. The results presented in Figure 2, show that $F E$ is a really noisy measure with overestimated values at low eccentricities, while $\epsilon$ is less noisy but depends on pore size. This is the case especially for pores with low eccentricity, when the signal difference is below the noise level. These findings are consistent with the original results presented in [30] which show noisy estimates in the grey matter of monkey brain. Moreover, both $F E$ and $\epsilon$ are underestimated when the acquisition protocol does not match the theoretical limit of long diffusion and mixing times. This analysis is focused on $F E$ and $\epsilon$, but the observations hold for similar indices presented in [29] which are based on signal differences.

In the model-based approach, we fit the diffusion signal itself, avoiding the increased noise variance of the signal difference. Moreover, we explicitly account for the finite gradient duration and we do not assume long mixing and diffusion times. Simulation 2 is focused on estimating microstructural parameters in isotropic substrates and we investigated the sensitivity of four different protocols: SDE, DDE $\|$, $\mathrm{DDE}_{\perp}$ and $\mathrm{DDE}_{\| \& \perp}$. For fair comparison, all protocols were adjusted to have the same maximum diffusion weighting (b-value) and number of measurements. First we test whether a simple model with identical pores (IFC) can provide good estimates of the volume-weighted mean pore size and eccentricity. In the elementary case when the underlying substrates have identical pores, the IFC model provides accurate parameter estimates. In this situation, DDE sequences offer no clear advantage over SDE sequences. This result is consistent with previous work which uses SDE measurements to estimate the parallel and perpendicular diffusivities, assuming 
an ensemble of identical micro-domains with time-independent diffusion coefficients $[51,52,53]$. However, this model underestimates radius and overestimates eccentricity if the substrates have a distribution of pore sizes for both types of protocols. Directly accounting for size distribution, i.e. the IGFC model, overcomes this problem and provides accurate microstructural estimates. The $\mathrm{DDE}_{\| \& \perp}$ protocol yields the smallest relative errors for all parameter estimates, with $50 \%$ of data points having a relative error less than $10 \%$ for mean radius and eccentricity estimates. From all IGFC model parameters, the shape of the gamma distribution $a$ has the largest variability which translates to larger interquartile range for the relative error of the size distribution variance compared to the other parameters, as illustrated in Table 2. Nevertheless, explicitly accounting for size distribution improves the accuracy of mean radius and eccentricity estimates. During fitting, $D$ was fixed to its ground truth value. For the protocols considered, additionally fitting for $D$ does not affect results significantly and provides good estimates of $D$, but increases the computational time, as the restricted signal depends on the ratio $D / R^{2}$.

When probing an ensemble of pores with a size distribution, the SDE protocol provides sensitivity to mean size and eccentricity, although the $\mathrm{DDE}_{\| \& \perp}$ and $\mathrm{DDE}_{\perp}$ protocols provide more accurate estimates. The DDE protocols yield smaller errors when estimating the variance of the size distribution, which is consistent with previous studies [32]. The sensitivity of the SDE protocol arises from the fact that choosing a specific model for parameter fitting resolves ambiguity in the underlying measurements. If the diffusion substrate is unknown, DDE measurements contain information to distinguish different cases. Moreover, a non model-based approach can be used to provide prior information in order to select a relevant model. If the appropriate model is assumed, then SDE measurements can estimate microstructural parameters. This is important in practice as the SDE sequence is much simpler to implement and generally returns higher signal by keeping the echo time shorter. Previous work [20] showed that DDE sequences preserve information from the diffusion-diffraction patterns in the presence of size distribution and can be used to recover more subtle features of pore shape, differentiating between various geometries [54, 55]. However, for estimating pore size and eccentricity in the diffusion regime considered here, the differences in performance among the protocols are quite small and further work is required to determine if they reflect genuine sensitivity differences. When comparing SDE and DDE sequences it is not trivial to define a fair comparison, and here we choose to have the same maximum diffusion weighting (b-value). Using the same maximum gradient strength would provide more diffusion weighting for DDE sequences, increasing sensitivity to small pore sizes. On the other and, the longer pulse duration for DDE reduces SNR if the effects of T2 decay are considered. A 
better comparison, which might well change the story, can be achieved if the protocols are optimised in order to fully explore the parameter space of each sequences, given practical constraints for total duration and gradient strength, as described in [46] or [56].

The microstructure models can be extended to estimate size and eccentricity in macroscopically anisotropic substrates. In Simulation 3 we used an extended protocol $\mathrm{DDE}_{R I}$ consisting of a SDE shell with 32 isotropic directions and DDE sequences with 6 gradient orientations (xx,xy,xz,yy,yz,zz). The SDE shell is used to determine the main orientation of the pores, which is subsequently fixed for fitting the rest of the model parameters. Fitting the WFC model to substrates with a size distribution, yields biased parameter estimates which underestimate mean radius and overestimate eccentricity. Accounting for size distribution, i.e. the WGFC model, provides accurate estimates of size and eccentricity, however an accurate value of the concentration parameter $\kappa$ is recovered only for pores with high eccentricity. For pores with low eccentricity, the influence of $\kappa$ on the overall diffusion signal is small, which is reflected in the poor estimate of this parameter at $E$ close to 1 .

In all the simulations discussed above, we used the same size distribution and orientation distribution for the cuboid meshes and the diffusion signal model. We overcome this limitation in Simulation 4. First, we show that the IGFC model can accurately recover pore size and eccentricity when the diffusion substrates have different size distributions. Thus we tested the cases of a parametric inverse Gaussian as well as a histologically plausible size distribution of cancer cells [48]. Moreover, we showed that the WGFC model can provide accurate microstructural parameter estimates when the diffusion substrate has a combination of coherently and randomly oriented pores.

In this study we constructed comprehensive diffusion protocols in order to have sensitivity over a wide range of diffusion substrates. Nevertheless, preliminary results (not shown) suggest that similar accuracy can be obtained with a much smaller number of measurements if protocols are tailored for a particular application. For example, we analyse an isotropic substrate with $\bar{l}_{x}=6 \mu \mathrm{m}, E=2, a=2.5$ and a subset of the $\mathrm{DDE}_{\| \& \perp}$ protocol with 25 measurements $(10$ different sequences with parallel and perpendicular gradients and $5 b=0$ measurements). We choose the measurements from the full protocol following a greedy algorithm to maximize sensitivity to this particular substrate. The parameter estimates from the short protocol overlap within standard deviation with the values obtained from the full protocol, however the estimates have higher variability (standard deviation over 10 noise trials is approximately twice larger). Thus, the right balance between acquisition time and measurement precision is necessary depending on the application. 
The models we propose describe a complex tissue architecture, however they include only intra-cuboidal diffusion. A first step towards practical applications, such as cancer imaging, where estimates of cellular eccentricity have the potential to distinguish between different tumour types [2], is to account for signal from the extracellular space. For low volume fractions, a simple extracellular model with hindered diffusion is accurate enough, while for higher volume fractions models that account for a time-dependent diffusivity which reflects the restriction length-scale [6] are preferable. Compartment models, as in $[1,9,10,11]$, can separate extracellular and intracellular contributions to the signal. The main challenge for testing such a model is creating a diffusion mesh with a realistic intracellular volume fraction around 0.6. Preliminary experiments, investigating an isotropic mesh with the intracuboidal space occupying around $15 \%$ of the total volume, show that we can estimate all microstructural parameters including volume fraction, but sensitivity reduces as volume fraction decreases.

The protocols used in this study provide sensitivity over a wide range of pores and are not designed for practical application. The maximum gradient strength we use in the simulation is $500 \sqrt{2} \mathrm{mT} / \mathrm{m}$ for SDE sequences and the maximum echo time required for the DDE protocols is $250 \mathrm{~ms}$. These values can be achieved on preclinical scanners, however, the range of values is more limited on a clinical scanner. The lower gradient strengths $(80 \mathrm{mT} / \mathrm{m}$ for a standard scanner or $300 \mathrm{mT} / \mathrm{m}$ for the Connectome scanner [57]) decrease the sensitivity to small pore size, while the short diffusion times affect the estimates for larger pores. The same features which are desired for such a simulation study (large measurement space, high gradient strength, long diffusion and mixing times) are a drawback for practical applications which require short acquisitions and limited diffusion time to prevent signal loss from $T 2$ decay. When designing such a protocol for practical applications all these aspects should be considered. Additionally, to preserve the sensitivity over a wide range of pore sizes for practical situations, rectangular gradients can be replaced with oscillating ones [56], or if there is prior knowledge of the system, the diffusion protocol can be substantially shortened using numerical optimisation [46], including diffusion gradients with varying orientation [58].

Future work will focus on more realistic substrates which include extracellular space with various volume fractions, in order to mimic different tissue configurations, as well as using improved measurement protocols which eventually could be used in practice. 


\section{Acknowledgements}

The EPSRC support this work through the following grants: EP/G007748, EP/H046410/01, EP/K020439/1, EP/M020533/1.

[1] E. Panagiotaki, S. Walker-Samuel, B. Siow, S. P. Johnson, V. Rajkumar, R. B. Pedley, M. F. Lythgoe, D. C. Alexander, Noninvasive quantification of solid tumor microstructure using VERDICT MRI, Cancer Research 74 (2014) 19021912.

[2] F. Szczepankiewicz, S. Lasič, D. van Westen, P. Sundgren, E. Englund, C. F. Westin, F. Ståhlberg, J. Lätt, D. Topgaard, M. Nilsson, Quantification of microscopic diffusion anisotropy disentangles effects of orientation dispersion from microstructure: applications in healthy volunteers and in brain tumors, NeuroImage 104 (2015) 241-52.

[3] P. Savadiev, J. S. W. Campbell, M. Descoteaux, R. Deriche, G. Bruce Pike, K. Siddiqi, Labeling of ambiguous subvoxel fibre bundle configurations in high angular resolution diffusion MRI, NeuroImage 41 (2008) 58-68.

[4] M. Nilsson, J. Lätt, F. Ståhlberg, D. van Westen, H. Hagslätt, The importance of axonal undulation in diffusion MR measurements: a monte carlo simulation study, NMR Biomed 25 (2012) 795-805.

[5] M. Kleinnijenhuis, V. Zerbi, B. Küsters, C. H. Slump, M. Barth, A. van Cappellen van Walsum, Layer-specific diffusion weighted imaging in human primary visual cortex in vitro, Cortex 25 (2013) 2569-82.

[6] D. S. Novikov, J. H. Jensen, J. A. Helpern, E. Fieremans, Revealing mesoscopic structural universality with diffusion, Proceedings of the National Academy of Sciences 11 (2014) 5088-5093.

[7] E. O. Stejskal, T. E. Tanner, Spin diffusion measurements: spin echoes in the presence of a time-dependent field gradient, Journal of Chemical Physics 42 (1965) 288-292.

[8] N. Shemesh, S. Jespersen, D. Alexander, Y. Cohen, I. Drobnjak, T. Dyrby, J. Finsterbusch, M. Koch, T. Kuder, F. Laun, M. Lawrenz, H. Lundell, P. Mitra, M. Nilsson, E. Özarslan, D. Topgaard, C. F. Westin, Conventions and nomenclature for double diffusion encoding (DDE) NMR and MRI, Magnetic Resonance in Medicine 75 (2016) 82-87. 
[9] Y. Assaf, T. Blumenfeld-Katzir, Y. Yovel, P. J. Basser, AxCaliber: a method for measuring axon diameter distribution from diffusion MRI, Magnetic Resonance in Medicine 59 (2008) 1347-1354.

[10] D. C. Alexander, P. L. Hubbard, M. G. Hall, E. A. Moore, M. Ptito, G. J. M. Parker, T. B. Dyrby, Orientationally invariant indices of axon diameter and density from diffusion MRI, NeuroImage 52 (2010) 1374-1389.

[11] U. Ferizi, T. Schneider, E. Panagiotaki, G. Nedjati-Gilani, H. Zhang, C. A. M. Wheeler-Kingshott, D. C. Alexander, A ranking of diffusion MRI compartment models with in vivo human brain data, Magnetic Resonance in Medicine 72 (2014) 1785-92.

[12] H. Zhang, P. L. Hubbard, G. J. M. Parker, D. C. Alexander, Axon diameter mapping in the presence of orientation dispersion with diffusion MRI, NeuroImage 56 (2011) 1301-1315.

[13] E. Özarslan, Compartment shape anisotropy (CSA) revealed by double pulsed field gradient MR, Journal of Magnetic Resonance 199 (2009) 56-67.

[14] D. G. Cory, A. N. Garroway, J. B. Miller, Applications of spin transport as a probe of local geometry, Polymer Preprints 31 (1990) 149-150.

[15] P. P. Mitra, Multiple wave-vector extensions of the NMR pulsed-field-gradient spin-echo diffusion measurement, Physical Review B 51(21) (1995) 15074-15078.

[16] M. E. Komlosh, E. Özarslan, M. J. Lizak, F. Horkay, V. Schram, N. Shemesh, Y. Cohen, P. J. Basser, Pore diameter mapping using double pulsed-field gradient MRI and its validation using a novel glass capillary array phantom, Journal of Magneic Resonance 208 (2011) 128-135.

[17] N. Shemesh, E. Özarslan, T. Adiri, P. J. Basser, Y. Cohen, Noninvasive bipolar double-pulsed-field-gradient NMR reveals signatures for pore size and shape in polydisperse, randomly oriented, inhomogeneous porous media, Journal of Chemical Physics 133.

[18] M. A. Koch, J. Finsterbusch, Compartment size estimation with double wave vector diffusion-weighted imaging, Magnetic Resonance in Medicine 60 (2008) 90-101. 
[19] M. A. Koch, J. Finsterbusch, Towards compartment size estimation in vivo based on double wave vector diffusion weighting, NMR in Biomedicine 24 (2011) 1422-32.

[20] N. Shemesh, E. Özarslan, P. J. Basser, Y. Cohen, Detecting diffusion-diffraction patterns in size distribution phantoms using double-pulsed field gradient NMR: Theory and experiments, Journal of Chemical Physics 132 (2010) 034703.

[21] P. T. Callaghan, I. Furo, Diffusion-diffusion correlation and exchange as a signature for local order and dynamics, Journal of Chemical Physics 120 (2004) 4032-4037.

[22] P. T. Callaghan, S. Godefroy, B. Ryland, Use of the second dimension in PGSE NMR studies of porous media, Magnetic Resonance Imaging 21 (2003) 243-248.

[23] M. A. Koch, J. Finsterbusch, Numerical simulation of double-wave vector experiments investigating diffusion in randomly oriented ellipsoidal pores, Magnetic Resonance in Medicine 62 (2009) 247-254.

[24] Y. Cheng, D. Cory, Multiple scattering by NMR, J. Am. Chem. Soc 121 (1999) 7935-7396.

[25] N. Shemesh, E. Özarslan, P. J. Basser, Y. Cohen, Accurate noninvasive measurement of cell size and compartment shape anisotropy in yeast cells using double-pulsed field gradient MR, NMR in Biomedicine 25 (2012) 236-246.

[26] N. Shemesh, D. Barazany, O. Sadan, L. Bar, Y. Zur, Y. Barhum, N. Sochen, D. Offen, Y. Assaf, Y. Cohen, Mapping apparent eccentricity and residual ensemble anisotropy in the gray matter using angular double-pulsed-field-gradient mri, Magnetic Resonance in Medicine 68 (2012) 795-806.

[27] N. Shemesh, J. T. Rosenberg, J. N. Dumez, J. A. Muniz, S. C. Grant, L. Frydman, Metabolic properties in stroked rats revealed by relaxation-enhanced magnetic resonance spectroscopy at ultrahigh fields, Nature Communications 5 (2014) 4958.

[28] A. V. Avram, E. Özarslan, J. E. Sarlls, P. J. Basser, In vivo detection of microscopic anisotropy using quadruple pulsed-field gradient (qPFG) diffusion MRI on a clinical scanner, NeuroImage 64 (2014) 229-239. 
[29] M. Lawrenz, M. A. Koch, J. Finsterbusch, A tensor model and measures of microscopic anisotropy for double-wave-vector diffusion-weighting experiments with long mixing times, Journal of Magnetic Resonance 202 (2010) 43-56.

[30] S. N. Jespersen, H. Lundell, C. K. Sonderby, T. B. Dyrby, Orientationally invariant metrics of apparent compartment eccentricity from double pulsed field gradient diffusion experiments, NMR in Biomedicine 26 (2013) 1647-1662.

[31] S. Lasič, F. Szczepankiewicz, S. Eriksson, M. Nilsson, D. Topgaard, Microanisotropy imaging: quantification of microscopic diffusion anisotropy and orientational order parameter by diffusion MRI with magic-angle spinning of the q-vector, Frontiers in Physics 2.

[32] D. Benjamini, M. E. Komlosh, P. J. Basser, U. Nevo, Nonparametric pore size distribution using d-PFG: Comparison to s-PFG and migration to MRI, Journal of Magnetic Resonance 246 (2014) 36-45.

[33] D. Benjamini, P. J. Basser, Joint radius-length distribution as a measure of anisotropic pore eccentricity: an experimental and analytical framework, Journal of Chemical Physics 141 (2014) 214202.

[34] M. G. Hall, D. C. Alexander, Convergence and parameter choice for MonteCarlo simulations of diffusion MRI, IEEE Trans. Medical Imaging 28 (2009) $1354-1364$.

[35] C. H. Neuman, Spin echo of spins diffusing in a bounded medium, Journal of Chemical Physics 60 (1974) 4508-4511.

[36] E. Özarslan, P. J. Besser, Microscopic anisotropy revealed by NMR double pulsed field gradient experiments with arbitrary timing parameters, Journal of Chemical Physics 128 (2009) 154511.

[37] S. N. Jespersen, N. Buhl, The displacement correlation tensor: Microstructure, ensemble anisotropy and curving fibers, Journal of Magnetic Resonance 208 (2011) 34-43.

[38] A. Ianus, B. Siow, I. Drobnjak, H. Zhang, D. C. Alexander, Gaussian phase distribution approximations for oscillating gradient spin-echo diffusion (MRI), Journal of Magnetic Resonance 227 (2013) 25-34. 
[39] Y. Assaf, R. Z. Freidlin, G. K. Rohde, P. J. Basser, New modeling and experimental framework to characterize hindered and restricted water diffusion in brain white matter, Magnetic Resonance in Medicine 52 (2004) 965-978.

[40] J. Stepisnik, Time-dependent self-diffusion by NMR spin echo, Physica B 183 (1993) 343-350.

[41] B. Balinov, B. Jonsson, P. Linse, O. Soderman, The NMR self-diffusion method applied to restricted diffusion. Simulation of echo attenuation from molecules in spheres and between planes, Journal of Magnetic Resonance. Series A. 104 (1993) 17-25.

[42] F. Aboitiz, A. B. Scheibel, R. S. Fisher, E. Zaidel, Fiber composition of the human corpus callosum, Brain Research 598 (1992) 143-153.

[43] J. C. Fiala, K. M. Harris, Dendrite Structures, Oxford University Press, 1999.

[44] G. Vona, A. Sabile, M. Louha, V. Sitruk, S. Romana, K. Schutze, F. Capron, D. Franco, M. Pazzagli, M. Vekemans, B. Lacour, C. Brechot, P. PaterliniBrechot, Isolation by size of epithelial tumor cells, American Journal of Pathology 156 (2000) 57-63.

[45] C. A. Baron, C. Beaulieu, Oscillating gradient spin-echo (OGSE) Diffusion Tensor Imaging of the human brain, Magnetic Resonance in Medicine 72 (2014) 726-736.

[46] D. C. Alexander, A general framework for experiment design in diffusion MRI and its application in measuring direct tissue-microstructure features, Magnetic Resonance in Medicine 60 (2008) 439-448.

[47] I. Drobnjak, B. Siow, D. C. Alexander, Optimizing gradient waveforms for microstructure sensitivity in diffusion-weighted MRI, Journal of Magnetic Resonance 206 (2010) 41-51.

[48] Nexelcom Bioscience LLC, (C)2003-2015. Analyzing NCI-60 Cancer Cell Lines, Available from: http://www.nexcelom.com/Applications/Cancer-Cells.html, [Accessed on 07.09.2015].

[49] T. B. Dyrby, L. V. Søgaard, M. G. Hall, M. Ptito, D. C. Alexander, Contrast and stability of the axon diameter index from microstructure imaging with diffusion MRI, Magnetic Resonance in Medicine 70 (2013) 711-721. 
[50] H. Zhang, T. Schneider, C. A. M. Wheeler-Kingshott, D. C. Alexander, NODDI: Practical in vivo neurite orientation dispersion and density imaging of the human brain, NeuroImage 61 (2012) 1000-1016.

[51] D. Topgaard, O. Söderman, Self-diffusion in two- and three-dimensional powders of anisotropic domains: An NMR study of the diffusion of water in cellulose and starch, The Journal of Physical Chemistry 106 (2002) 11887 - 92.

[52] F. Stallmach, J. Kärger, C. Krause, M. Jeschke, U. Oberhagemann, Evidence of anisotropic self-diffusion of guest molecules in nanoporous materials of MCM-41 type, Journal of the American Chemical Society 122 (2000) 9237-9242.

[53] E. Kaden, F. Kruggel, D. C. Alexander, Quantitative mapping of the per-axon diffusion coefficients in brain white matter, Magnetic Resonance in Medicine Early View.

[54] N. Shemesh, C.-F. Westin, Y. Cohen, Magnetic resonance imaging by synergistic diffusion-diffraction patterns, Physical Review Letters 108 (2012) 058103.

[55] T. A. Kuder, F. B. Laun, NMR-based diffusion pore imaging by double wave vector measurements., Magnetic Resonance in Medicine 70 (2013) 836-841.

[56] I. Drobnjak, H. Zhang, A. Ianuş, E. Kaden, D. C. Alexander, PGSE, OGSE, and sensitivity to axon diameter in diffusion mri: Insight from a simulation study, Magnetic Resonance in Medicine Early view.

[57] J. A. McNab, B. L. Edlow, T. Witzel, S. Y. Huang, H. Bhat, K. Heberlein, T. Feiweier, K. Liu, B. Keil, J. Cohen-Adad, M. D. Tisdal, R. Folkerth, H. C. Kinney, L. L. Wald, The human connectome project and beyond: initial applications of $300 \mathrm{mt} / \mathrm{m}$ gradients, NeuroImage 80 (2013) 234-45.

[58] I. Drobnjak, D. C. Alexander, Optimising time-varying gradient orientation for microstructure sensitivity in diffusion-weighted MR, Journal of Magnetic Resonance 212 (2011) 344-354. 